\title{
Efficacy and safety of dual-polymer hydroxypropyl guar- and hyaluronic acid-containing lubricant eyedrops for the management of dry-eye disease: a randomized double-masked clinical study
}

This article was published in the following Dove Press journal: Clinical Ophthalmology

\author{
Marc Labetoulle' \\ Stefanie Schmickler ${ }^{2}$ \\ David Galarreta ${ }^{3}$ \\ Daniel Böhringer ${ }^{4}$ \\ Abayomi Ogundele ${ }^{5}$ \\ Michel Guillon ${ }^{6}$ \\ Christophe Baudouin 7,8 \\ 'Ophtalmologie, Hôpital Bicêtre, \\ APHP, South Paris Université, \\ Kremlin-Bicêtre, Paris, France; ${ }^{2}$ Augen- \\ Zentrum-Nordwest Augenpraxis, \\ Ahaus, Germany; ${ }^{3}$ Hospital Clinico \\ Universitario de Valladolid, Valladolid, \\ Spain; ${ }^{4}$ Eye Center, University Hospital \\ Freiburg, Freiburg, Germany; ${ }^{5}$ Novartis \\ Pharmaceutical Corporation, Fort \\ Worth, TX, USA; ${ }^{6}$ Ocular Technology \\ Group, London, UK; ${ }^{7}$ Quinze-Vingts \\ Hospital, DHU Sight Restore, \\ University Versailles Saint Quentin en \\ Yvelines, INSERM-DHOS CIC, Paris, \\ France; ${ }^{8}$ Sorbonne Universités, UPMC \\ Univ Paris 06, INSERM, CNRS, Institut \\ de la Vision, Paris, France
}

Correspondence: Marc Labetoulle Ophtalmologie, Hôpital Bicêtre, APHP, South Paris Université, 78 Rue du Général Leclerc, Kremlin-Bicêtre, Paris, France

Tel +33 I6 9823840

Email marc.labetoulle@aphp.fr
Background: An artificial-tear formulation containing the dual polymers hydroxypropyl guar (HPG) and hyaluronic acid (HA) is approved for the treatment of dry-eye disease (DED). The present study compared the efficacy and safety of the HPG-HA dual-polymer formulation vs a sodium hyaluronate ( $\mathrm{SH}$ )-containing artificial-tear formulation in patients with DED.

Methods: In a prospective, 6-week, multicenter, double-masked, parallel-group study, patients with DED aged $\geq 18$ years and total ocular surface staining (TOSS) score $\geq 4$ and $\leq 9$ were randomized (1:1) to receive either HPG-HA or SH four times a day for 42 days. Changes from baseline in TOSS (primary end point), impact of dry eye on everyday life (IDEEL) treatment-satisfaction scores (effectiveness and inconvenience), and tear-film breakup time (TFBUT) at day 42 were assessed using a fixed-sequence testing strategy. Noninferiority was assessed on the primary end point based on the upper limit of two-sided 95\% CIs for mean treatment difference (HPG-HA or SH) $<2$ units.

Results: In total, 99 patients were randomized (HPG-HA, $n=50 ; \mathrm{SH}, \mathrm{n}=49$ ). At day 42, the least square (LS) mean \pm SE change from baseline in TOSS was $-1.16 \pm 0.24$ and $-0.92 \pm 0.23$ in the HPG-HA and $\mathrm{SH}$ groups, respectively, and the treatment difference was $-0.24 \pm 0.33$ ( $95 \% \mathrm{CI}-0.90$ to 0.42$)$. Noninferiority was demonstrated as the upper limit of the $95 \%$ CI was $<2$ units. LS mean change from baseline at day 42 for HPG-HA vs SH was -3.18 ( $P=0.4817)$ in IDEEL treatment-effectiveness scores, -12.56 $(P=0.0001)$ in treatment-inconvenience scores, and 0.30 seconds $(P=0.5789)$ in TFBUT.

Conclusion: The HPG-HA dual-polymer formulation was noninferior to the SH lubricant eyedrops for improvement in ocular surface staining in DED. HPG-HA did not show improvement over SH in IDEEL treatment-satisfaction scores. No new safety findings were reported.

Keywords: dry-eye disease, dual polymer, artificial tears, hydroxypropyl guar, hyaluronic acid

\section{Abbreviations}

AE, adverse event; BCVA, best-corrected visual acuity; DED, dry-eye disease; HA, hyaluronic acid; HPG, hydroxypropyl guar; IDEEL, impact of dry eye on everyday life; ITT, intent to treat; LS, least squares; MMRM, mixed-model repeated measure; PEG, polyethylene glycol; PG, propylene glycol; QID, quater in die (four times a day); $\mathrm{SH}$, sodium hyaluronate; TFBUT, tear-film breakup time; TOSS, total ocular surface staining; UL, upper limit.

\section{Background}

Dry-eye disease (DED) is a chronic condition caused by either excessive evaporation or deficiency of the tear film that leads to tear hyperosmolarity and inflammation 
of the ocular surface. ${ }^{1}$ Artificial tears are the mainstay for management of DED, and depending on the severity of the condition, are used either alone or concomitantly with other treatments. ${ }^{2-4}$ Artificial tears are mostly buffered hypotonic or isotonic solutions that supplement tear-film volume and stability and lubricate the ocular surface, thus protecting it against desiccation. ${ }^{3,4}$ Several types of artificial tears are available that differ mostly in terms of the composition of polymers, electrolyte, osmolarity, solutes, and the presence or absence of preservatives. . $^{2,4}$

Polymers are an important constituent of artificial tears, as they determine the viscosity of the formulation and aid in tear-film stabilization by increasing the retention time of the lubricant on the ocular surface and thus enhancing the duration of comfort and quality of vision. ${ }^{2,4,6}$ Hydroxypropyl guar (HPG) is a gellable agent with mucomimetic properties that has been shown to reduce surface friction and possesses rheological properties very similar to those of tears. ${ }^{7}$ Several clinical studies have demonstrated the effectiveness of HPGcontaining artificial tears in the management of DED. ${ }^{7-12}$ Hyaluronic acid (HA), an anionic mucopolysaccharide, is the key component of the extracellular matrix and plays a critical role in cell proliferation, anti-inflammation, and wound repair. ${ }^{13,14}$ Because of its viscoelastic and hygroscopic properties, HA is widely used in tear-substitute formulations. ${ }^{15,16}$ Studies have shown that sodium hyaluronate-based lubricant eyedrops help improve both signs and symptoms in patients with DED. ${ }^{17-21}$

Artificial tears that contain a combination of polymers are more likely to provide better ocular lubrication, retention, and synergistic benefits to the ocular surface than single-polymer-based artificial tears. Systane hydration (Alcon Laboratories, Fort Worth, TX, USA; HPG-HA) is an artificial-tear formulation that contains HPG, HA, and the demulcents propylene glycol (PG) and polyethylene glycol (PEG), and is approved for the treatment of DED. The purpose of this study was to demonstrate the noninferiority of HPG-HA artificial tears to sodium hyaluronate (SH) containing artificial-tear formulation (Hyabak 0.15\%; Laboratoires Théa, Clermont-Ferrand, France) in patients with DED.

\section{Methods}

This was a prospective, 6-week, multicenter, doublemasked, parallel-group, randomized, noninferiority study conducted at ten centers across France, Germany, Spain, and the UK from July 2015 to May 2016, and is registered at ClinicalTrials.gov (NCT02470429). The study consisted of an open-label run-in period followed by a 6-week, doublemasked treatment phase. During the run-in-period, eligible patients self-administered one drop of preservative-free $0.9 \%$ saline eyedrops in both eyes four times a day (QID [quater in die]) for 7-14 days (Figure 1). On day 0 (baseline visit), patients who qualified through reevaluation assessment (ie, had a total ocular surface staining score [TOSS] $\geq 4$ and $\leq 9$ ), were randomized 1:1 to receive either HPG-HA or SH lubricant eyedrops, with one drop in each eye instilled QID for 42 days (Figure 1).

One eye from each patient was selected as the study eye. If both eyes met the inclusion criteria, the worse eye (defined as the eye with greater TOSS at the screening visit) was selected as the study eye. If both eyes had the same TOSS at the screening visit, the right eye was selected as

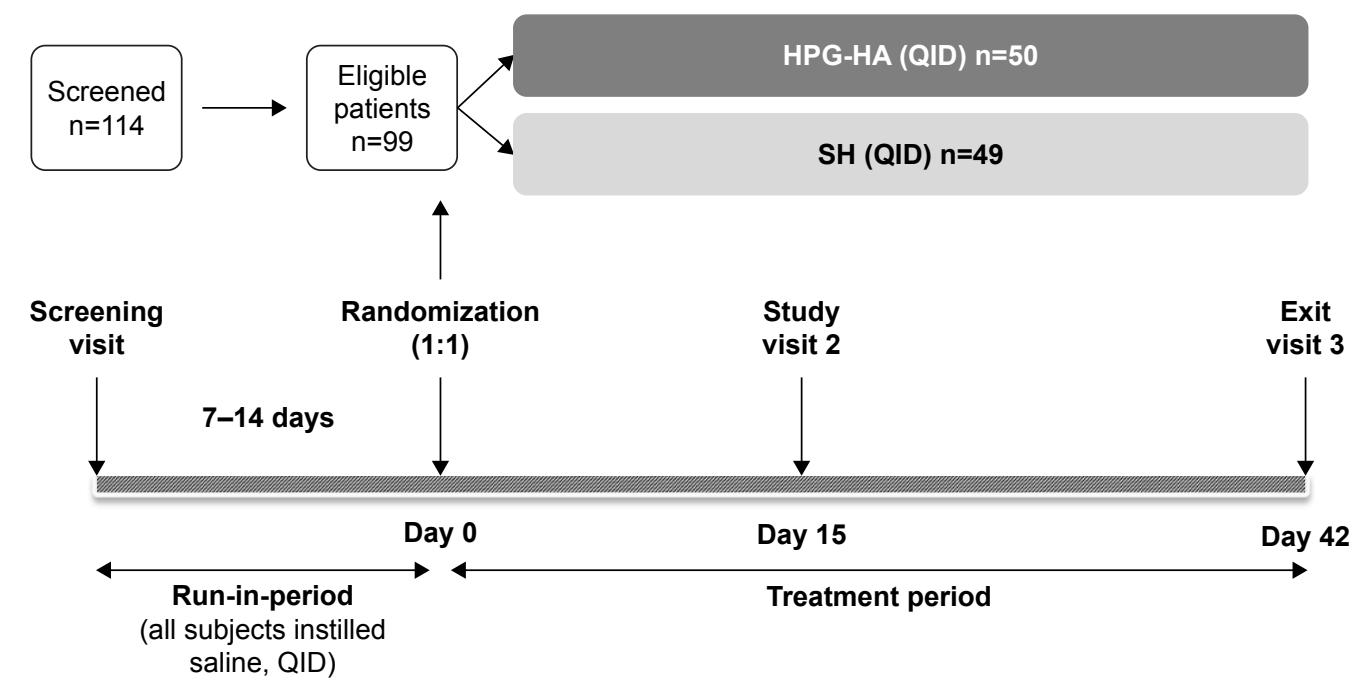

Figure I Study design.

Abbreviations: HPG-HA, hydroxypropyl guar-hyaluronic acid; QID, quater in die (four times a day); SH, sodium hyaluronate. 
the study eye. Patients were randomized sequentially in the study, and the randomization schedule was blocked to ensure balance of treatment allocations within each study center. Randomization was implemented using an interactive response technology system. All patients provided written informed consent before enrollment in the study.

The study protocol was approved by the central institutional review board of each participating country (Ethik-Kommission der Germany; CEIC IDC Salud a Cataluña, Spain; CPP Ile de France 3, France; CEIC Area de Salud de Valladolid Este, Spain; NRES Committee East of England, Cambridge, UK), and in addition by two local institutional review boards in Germany (Ethik-Kommission bei der Aerztekammer der Universitaet Münster and Ethik-Kommission bei der Aerztekammer des Saarlandes). The study was conducted in accordance with the Declaration of Helsinki and good clinical practice, and was in compliance with all federal, local, or regional requirements.

\section{Lubricant-eyedrop composition}

Systane hydration is a sterile solution containing HPG, propylene glycol, PEG, $0.15 \% \mathrm{SH}$, sorbitol, aminomethylpropanol, boric acid, sodium borate, disodium EDTA, sodium citrate, potassium chloride, and sodium chloride preserved with $0.001 \%$ Polyquad (polidronium chloride). Hyabak $0.15 \%$ is a preservative-free, sterile solution containing $0.15 \% \mathrm{SH}$, sodium chloride, tromethamine, hydrochloric acid, and water.

\section{Patients}

Eligible patients were $\geq 18$ years of age, had diagnosed DED for at least 3 months prior to screening, had TOSS 4-9 on the 15-point Oxford scale in one eye at screening, had unanesthetized Schirmer I test score 3-9 $\mathrm{mm}$ and/or the sum of three measurements of tear-film breakup time $($ TFBUT $) \leq 15$ seconds in that same eye, and had been using non-benzalkonium chloride-containing artificial tears at least once a day for at least 3 months prior to screening.

Patients who had best-corrected visual acuity (BCVA) of $\leq 55$ Early Treatment Diabetic Retinopathy Study letters in each eye at screening; were hypersensitive to any study product or any excipients; had a history of ocular or intraocular surgery in either eye or serious ocular trauma in either eye within 6 months prior to the screening visit; had an active ocular infection or inflammation not associated with dry eye at screening and randomization visits; had participated in any other investigational clinical study within 30 days prior to screening in this study; were taking chronic systemic medication (including prescription therapies, over-the-counter therapies, and vitamins/supplements) and had been on a stable dose for $<30$ days prior to the screening visit; had any anticipated change in dosing regimen during the course of the study; or had any uncontrolled systemic disease were not eligible to participate in the study. Female patients who were breastfeeding or pregnant or who had a positive pregnancy test at screening were not considered for enrollment.

\section{Study end points}

The primary end point was change from baseline in TOSS at day 42 . Secondary end points were change from baseline at day 42 in: treatment-satisfaction scores (treatment effectiveness and treatment inconvenience) based on the impact of dry eye on everyday life (IDEEL) treatment-satisfaction module, TFBUT, and TOSS (test for superiority). Exploratory objectives included comparison of tear-film kinetic measures, conjunctival staining improvements, and tear-film osmolarity in patients after 42 days of QID dosing with HPG-HA and $\mathrm{SH}$ eyedrops. Safety evaluations included adverse events (AEs), BCVA, and ocular signs.

\section{Assessments}

TOSS, TFBUT, tear-film osmolarity, tear-film kinetics, and objective bulbar conjunctival staining were assessed at screening, day 0 (baseline), day 15, and day 42 (exit) study visits. Details regarding assessment procedures are provided in Supplementary material. TOSS was evaluated in both eyes, and the right eye was assessed first. The investigators used the Oxford grading scheme to quantify the degree of staining observed and specifically compared the appearance of staining on exposed corneal and interpalpebral conjunctivae using a visual diagram. The visual diagram was used to correlate the visual element with a numerical grade that ranged from 0 (absent staining) to 5 (severe staining). Grades for the amount of staining observed in the corneal, nasal-conjunctival, and temporal-conjunctival regions of each eye were recorded and summed to generate the TOSS for each eye, which ranged $0-15$.

The IDEEL treatment-satisfaction questionnaire was completed at days 0,15 , and 42 and comprised ten items that assessed each patient's general satisfaction with their treatment over a 2-week period (Supplementary material). For all items, patients were instructed to select a single response (0-4). The first IDEEL score was calculated as the mean response to questions $2-5$, with the result multiplied by 25 . The second IDEEL score was then calculated as the mean response to questions $6,8,9$, and 10 , with the 
result multiplied by 25 . Both the first and second IDEEL scores were recorded. Responses to questions 1 and 7 were not included in score computations. The possible range of each IDEEL score was 0 (complete disability) to 100 (no disability). TFBUT was assessed using a sodium fluorescein strip (Supplementary material). The assessment was conducted three times for each eye, and each TFBUT was measured in seconds. The average of the three measurements was recorded as the final TFBUT for each visit.

Tear-osmolarity measurements were obtained using TearLab ${ }^{\mathrm{TM}}$ osmolarity readers, pens, and test cards. Tearfilm kinetic measures and objective bulbar conjunctival staining assessments were conducted only at selected study centers that had the necessary capabilities. All AEs reported before the initiation of study treatment were classified as pretreatment AEs, and all AEs with an onset after study-treatment initiation and up to discontinuation of study treatment (ie, $42 \pm 7$ days) were classified as treatment-emergent AEs.

\section{Statistical analysis}

Statistical analyses were performed using SAS software (version 9.2; SAS Institute, Cary, NC, USA). With at least 40 evaluable patients per group, the study had $94 \%$ power to demonstrate noninferiority of HPG-HA to SH by using a noninferiority margin of 2 units and assuming an expected difference of 0 units and an SD of 2.5 units. The primary end point was analyzed using the mixed-model repeatedmeasure method (MMRM). The model included terms for baseline assessments, treatment, visit, and treatmentvisit interaction. An unstructured variance-covariance matrix was used to model within-subject correlations. If the unstructured variance-covariance matrix resulted in a lack of convergence, then other covariance structures were investigated. Noninferiority was established if the upper limit (UL) of two-sided 95\% CIs for the least-square (LS) mean difference in TOSS between HPG-HA minus SH was $<2$.

Evaluation of secondary end points was also performed using the MMRM method, similar to the primary analysis. For IDEEL scores and TFBUT, improvement was concluded if the $P$-value was $<0.05$ for a positive LS mean difference between HPG-HA and SH. For TOSS at day 42, superiority was concluded if the $P$-value was $<0.05$ for a negative LS mean difference between HPG-HA and SH. Exploratory end points and safety variables were analyzed descriptively.

A fixed-sequence testing strategy was employed to ensure that the type I error rate was controlled at a 5\% level of significance (two-sided). Testing of a hypothesis required that all previous hypotheses be statistically significant. If the primary objective was met with statistical significance, only then was testing for secondary variables performed following a prespecified sequential order: IDEEL treatment-satisfaction scores at day 42, TFBUT at day 42, and TOSS at day 42. All efficacy evaluations were performed on the intent-to-treat set, which included all randomized patients who had received at least one dose of the study treatment. Only patients with day 42 tear-film kinetic measures, objective bulbar conjunctival staining scores, or tear osmolarity (as appropriate for the end point) were included in the exploratory efficacy analyses. Safety analyses were conducted using the safety-analysis set, which included all patients who had been exposed to the study product, including the run-in period.

\section{Results}

In total, 114 patients were screened, and 99 were randomized to the two treatment groups (HPG-HA, $n=50 ; H A, n=49$ ), and 97 completed the study. Two patients in the HPG-HA group discontinued the study (one due to an $\mathrm{AE}$ and other due to withdrawal of consent). All randomized patients were included in the intent-to-treat and safety analyses. The mean age of patients was $59.2 \pm 13.5$ years, and majority of patients were female (Table 1). IDEEL treatment-satisfaction scores, TFBUT, and TOSS at baseline in the HPG-HA and SH groups are summarized in Table 2. Baseline disease characteristics of patients were similar, with no meaningful differences between the two treatment groups.

\section{Primary efficacy outcome}

Both treatments led to a reduction in mean TOSS from baseline at day 42 (Table 2). The LS mean \pm SE change from baseline at day 42 in the TOSS was higher in the HPG-HA

Table I Demographic characteristics of patients overall and by treatment group (ITT set)

\begin{tabular}{l|l|l|l}
\hline & $\begin{array}{l}\text { HPG-HA } \\
(\mathbf{n}=\mathbf{5 0})\end{array}$ & $\begin{array}{l}\text { SH } \\
(\mathbf{n}=\mathbf{4 9})\end{array}$ & $\begin{array}{l}\text { Overall } \\
(\mathbf{n}=\mathbf{9 9})\end{array}$ \\
\hline $\begin{array}{l}\text { Mean age, years (SD) } \\
\begin{array}{l}\text { Age-group, n (\%) } \\
\text { (years) }\end{array}\end{array}$ & $61.7(12.29)$ & $56.7(14.29)$ & $59.2(13.49)$ \\
I8-64 & $30(60.0)$ & $31(63.3)$ & $6 I(61.6)$ \\
$\geq 65$ & $20(40.0)$ & $18(36.7)$ & $38(38.4)$ \\
Sex & $4 I(82.0)$ & $38(77.6)$ & $79(79.8)$ \\
$\begin{array}{l}\text { Female, n (\%) } \\
\text { Race, n (\%) }\end{array}$ & $42(84.0)$ & $38(77.6)$ & $80(80.8)$ \\
White & $3(6.0)$ & $5(10.2)$ & $8(8.1)$ \\
Black/African-American & $3(6.0)$ & 0 & $3(3.0)$ \\
Asian & $2(4.0)$ & $6(12.2)$ & $8(8.1)$ \\
Other &
\end{tabular}

Abbreviations: HPG-HA, hydroxypropyl guar-hyaluronic acid; ITT, intent to treat; $\mathrm{SH}$, sodium hyaluronate; SD, standard deviation. 
Table 2 Descriptive statistics of TOSS, IDEEL treatmentsatisfaction scores (effectiveness and inconvenience), and TFBUT at baseline and each study visit (ITT set)

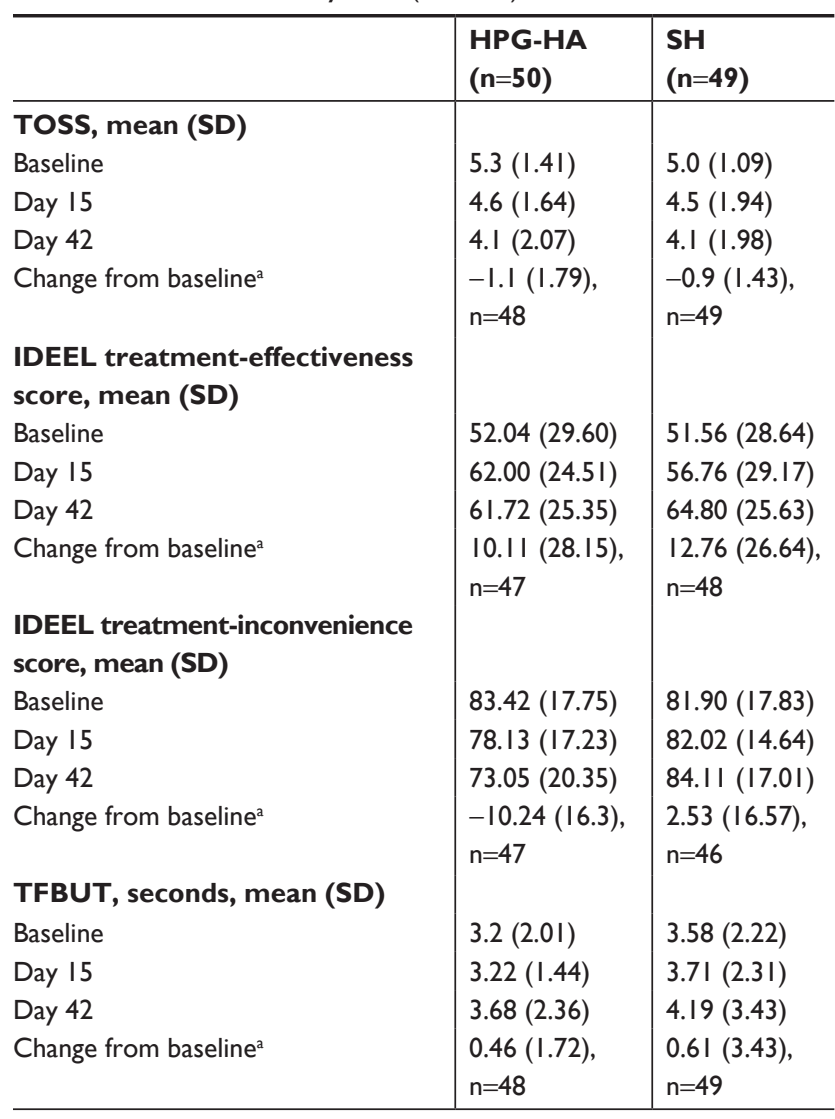

Notes: a Change from baseline to day 42 , calculated for each patient available at day 42 visit and then summarized. If a patient did not attend the visit or did not have the measurement taken (at baseline or day 42), then they were excluded from baseline calculation. IDEEL treatment-satisfaction scores range 0-100. TOSS is calculated from three staining areas of the ocular surface: corneal, nasal conjunctival, and temporal conjunctival. Each area is scored 0-5, with total patient scores of 0-I5.

Abbreviations: HPG-HA, hydroxypropyl guar-hyaluronic acid; IDEEL, impact of dry eye on everyday life; ITT, intent to treat; $\mathrm{SH}$, sodium hyaluronate; SD, standard deviation; TFBUT, tear-film breakup time; TOSS, total ocular surface staining.

group ( $-1.16 \pm 0.24)$ than the SH group $(-0.92 \pm 0.23)$. The LS mean \pm SE treatment difference was -0.24 (0.33), and the UL of $95 \%$ CI was 0.42 (Figure 2). Noninferiority of HPG-HA to SH lubricant eyedrops was demonstrated: UL of the $95 \%$ $\mathrm{CI}$ for the treatment difference between the groups $<2$.

\section{Secondary efficacy outcomes}

An increase in mean IDEEL treatment-effectiveness scores from baseline was observed in both treatment groups at day 42. However, HPG-HA did not show improvement over $\mathrm{SH}$ eyedrops for change in IDEEL treatment-effectiveness scores from baseline at day 42 (LS mean difference -3.18 units, $P=0.4817$; Figure 3). IDEEL treatment-inconvenience scores were lower with HPG-HA than SH eyedrops, and the difference between the groups was notably in favor of the

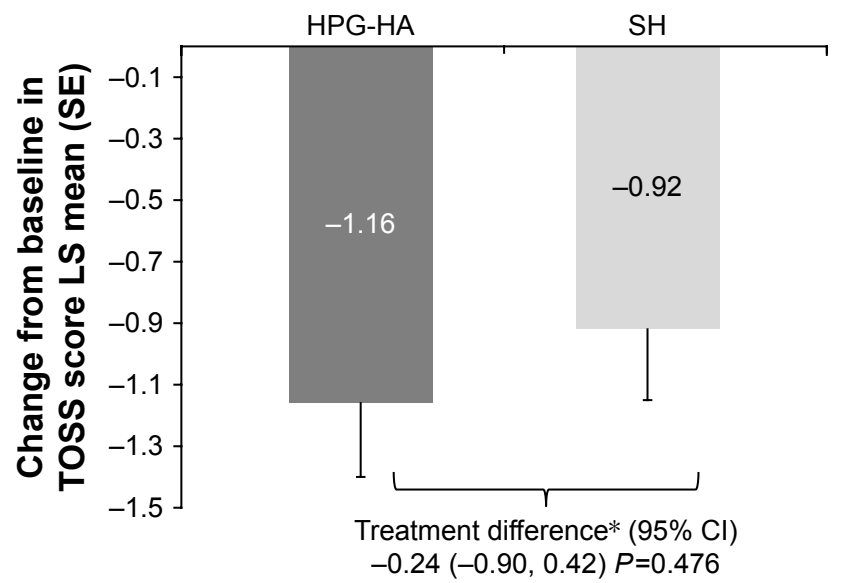

Figure 2 Change from baseline at day 42 in TOSS by treatment group (ITT set). Note: *Noninferiority of HPG-HA to SH lubricant eyedrops was demonstrated ( $\mathrm{UL}$ of $95 \% \mathrm{Cl}$ for the treatment difference between the groups was $<2$ ). Abbreviations: HPG-HA, hydroxypropyl guar-hyaluronic acid; ITT, intent to treat; LS, least square; SE, standard error; SH, sodium hyaluronate; TOSS, total ocular surface staining; UL, upper limit.

single-polymer formulation (LS mean difference -12.56 units, $P=0.0001$; Figure 3). Thus, the study did not meet its first secondary objective. HPG-HA did not show any improvement over SH eyedrops for mean change from baseline in TFBUT (LS mean difference, -0.30 units; $P=0.5789$; Figure 4). While there was a nominal treatment difference in change from baseline in TOSS at day 42 in favor of HPG-HA, superiority of HPG-HA over SH was not established (LS mean difference $-0.24, P=0.4760$; Figure 2).

\section{Exploratory end points}

Tear-film kinetic measurements were conducted only in a subset of 30 patients (HPG-HA, $n=17 ; \mathrm{SH}, \mathrm{n}=13$ ) at selected study centers. Overall, no clinically meaningful differences were observed between the HPG-HA- and SH-treated patients for change from baseline at day 42 in tear-film kinetic measurements (percentage of protective area, dehydration speed, noninvasive breakup time, interblink period, percentage of exposed area at first break, and percentage of exposed area at blink), objective bulbar conjunctival staining score (LS mean difference $-0.01, P=0.9459$ ) and tear osmolarity (LS mean difference $0.78, P=0.7328$ ).

\section{Safety}

Overall, $18.0 \%(n=9)$ and $20.4 \%(n=10)$ of patients reported at least one treatment-emergent ocular AE in the HPG-HA and $\mathrm{SH}$ groups, respectively. Of these, in $14.0 \%(n=7)$ of patients in the HPG-HA group and in $10.2 \%(n=5)$ of patients in the SH groups, ocular AEs were considered to be treatment-related. All treatment-emergent ocular AEs 


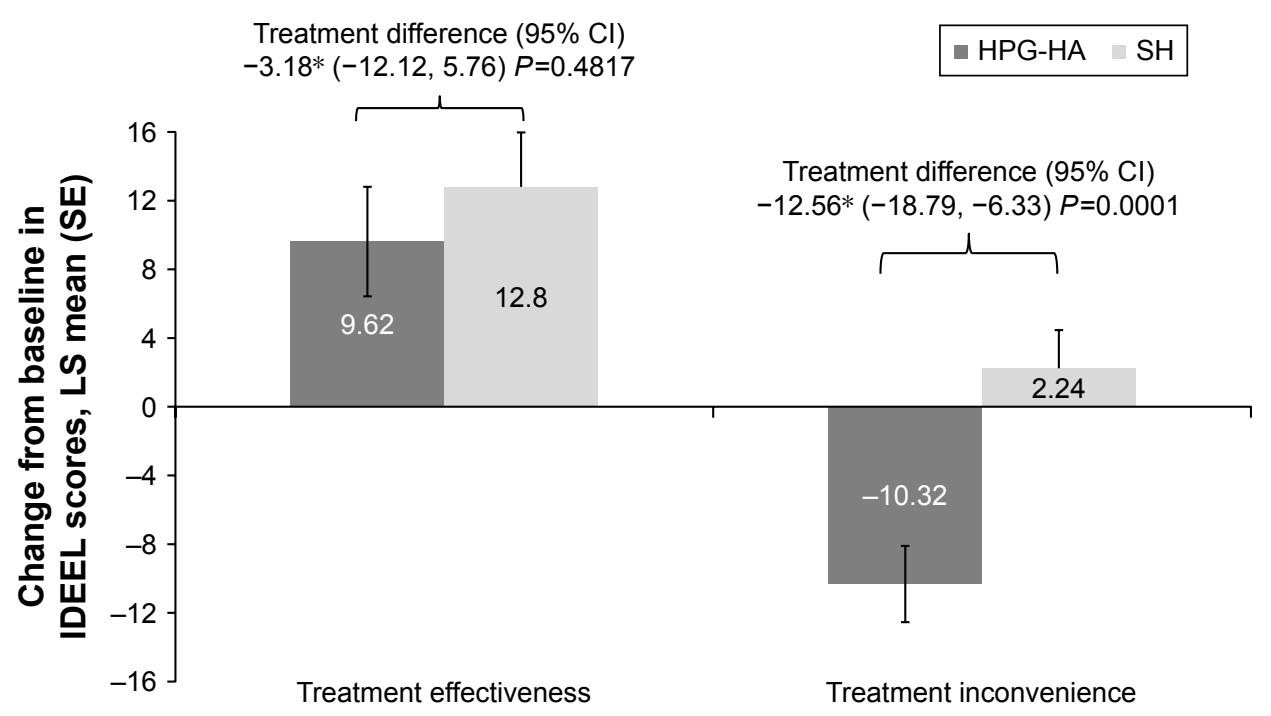

Figure 3 Change from baseline at day 42 in IDEEL treatment-satisfaction scores (effectiveness and inconvenience) by treatment group (ITT set). Note: *A positive difference would have favored HPG-HA.

Abbreviations: HPG-HA, hydroxypropyl guar-hyaluronic acid; IDEEL, impact of dry eye on everyday life; ITT, intent to treat; LS, least square; SE, standard error; SH, sodium hyaluronate.

are summarized in Table 3. All ocular AEs were classified as mild-moderate in severity.

In the HPG-HA group, the reported treatment-related ocular AEs were blurred vision (10\%), eye irritation ( $8 \%$ ), abnormal sensation in the eye $(2 \%)$, and reduced visual acuity (2\%). In the SH group, eye irritation (4.1\%) was the only treatment-related ocular AE reported by more than one patient. Other treatment-related ocular AEs (2\%) reported in the $\mathrm{SH}$ group were abnormal sensation in the eye, eyelid edema, dry eye, foreign-body sensation in the eye, lacrimal disorder, ocular hyperemia, and reduced visual acuity (one patient each).

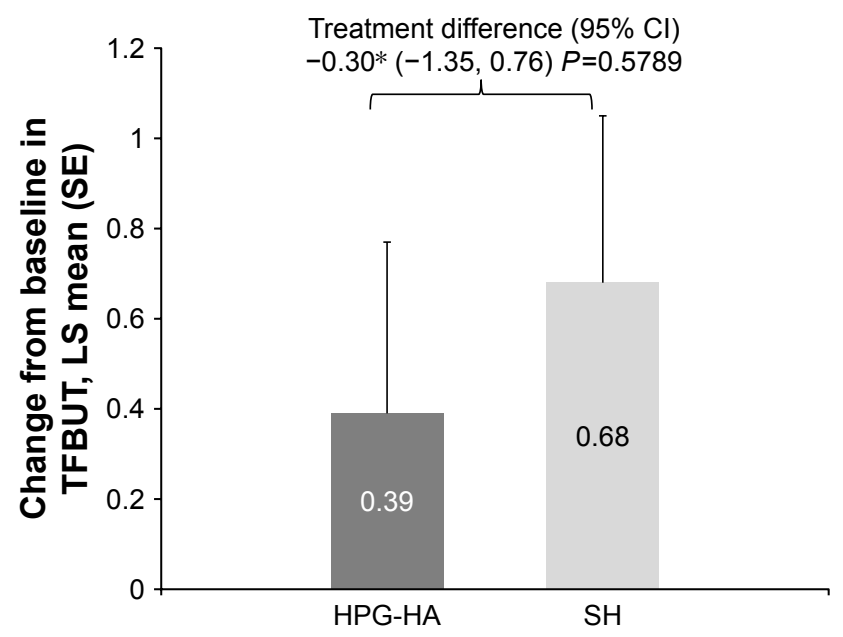

Figure 4 Change from baseline at day 42 in TFBUT by treatment group (ITT set). Note: *A positive difference would have favored HPG-HA.

Abbreviations: HPG-HA, hydroxypropyl guar-hyaluronic acid; ITT, intent to treat; LS, least square; SE, standard error; SH, sodium hyaluronate; TFBUT, tearfilm breakup time.
Nonocular AEs were reported in $18.0 \%(n=9)$ and $12.2 \%$ $(n=6)$ of patients in the HPG-HA and SH groups, respectively. None of the nonocular AEs was considered treatment-related. Two nonocular serious AEs were reported, one each in the HPG-HA (cholelithiasis) and SH (intervertebral disk operation) groups. Neither serious AE was considered treatmentrelated. One patient in the HPG-HA group discontinued

Table 3 Most frequently (incidence $\geq 1 \%$ ) reported ocular treatment-emergent adverse events by system-organ class and preferred term in each treatment group (safety set)

\begin{tabular}{l|l|l}
\hline & $\begin{array}{l}\text { HPG-HA } \\
(\mathbf{n}=\mathbf{5 0 )}\end{array}$ & $\begin{array}{l}\mathbf{S H} \\
\mathbf{( n = 4 9 )}\end{array}$ \\
\hline Eye disorders, $\mathbf{n}(\%)$ & $9(I 8.0)$ & $\mathrm{I} 0(20.4)$ \\
Abnormal sensation in eye & $2(4.0)$ & $\mathrm{I}(2.0)$ \\
Dry eye & 0 & $\mathrm{I}(2.0)$ \\
Eye irritation & $5(10.0)$ & $3(6.1)$ \\
Eye pain & 0 & $\mathrm{I}(2.0)$ \\
Eyelid edema & 0 & $\mathrm{I}(2.0)$ \\
Foreign-body sensation in eyes & 0 & $2(4.1)$ \\
Halo vision & $\mathrm{I}(2.0)$ & 0 \\
Lacrimal disorder & 0 & $\mathrm{I}(2.0)$ \\
Meibomian gland dysfunction & 0 & $\mathrm{I}(2.0)$ \\
Ocular hyperemia & 0 & $\mathrm{I}(2.0)$ \\
Ocular jaundice & 0 & $\mathrm{I}(2.0)$ \\
Vision blurred & $5(I 0.0)$ & $\mathrm{I}(2.0)$ \\
Visual acuity reduced & $\mathrm{I}(2.0)$ & $2(4.1)$ \\
Nervous system disorders, $\mathbf{n}(\%)$ & $\mathrm{I}(2.0)$ & 0 \\
Burning sensation & $\mathrm{I}(2.0)$ & 0 \\
Surgical and medical procedures, $\mathbf{n}(\%)$ & 0 & $\mathrm{I}(2.0)$ \\
Eyelid operation & 0 & $\mathrm{I}(2.0)$ \\
\hline
\end{tabular}

Note: Adverse events coded using MedDRA version 18.0.

Abbreviations: HPG-HA, hydroxypropyl guar-hyaluronic acid; SH, sodium hyaluronate. 
the study due to a nonocular AE (arthralgia). This AE was not considered by the investigator to be treatment-related. There were no deaths during the study. On ocular examinations (BCVA and slit-lamp biomicroscopy), no meaningful differences were observed between the HPG-HA and SH groups. For most patients in both treatment groups, scores at baseline for slit-lamp parameters of aqueous flare, aqueous cells, lens, and lens status remained unchanged at each postbaseline study visit.

\section{Discussion}

This was the first multi-dose clinical study to evaluate the efficacy and safety of dual-polymer HPG-HA-containing artificial tears in patients with DED. The study demonstrated the noninferiority of HPG-HA- to SH-containing artificial tears in reducing clinical signs of dry eye in patients with DED, as indicated by reduction in TOSS from baseline to day 42. Our results suggest that HPG-HA has at least similar effectiveness as $\mathrm{SH}$ eyedrops, which are recommended to moisten and lubricate the eye in cases of dryness sensations or ocular fatigue induced by external factors.

Both HA and HPG used individually in artificial tears have been demonstrated to improve symptoms in patients with DED. HA has extensive water-retention capacity and provides sustained hydration, increases residence time of lubricating drops by decreasing the tear drainage rate and enhancing tear film stability, and is known to accelerate corneal epithelial wound healing. ${ }^{13-16,22} \mathrm{HPG}$ is a $\mathrm{pH}$-sensitive molecule that exhibits low viscosity in solutions at $\mathrm{pH} \leq 7$, but forms a thin cross-linked gel-like matrix with borate ions when exposed to the tear film $(\mathrm{pH} 7.5)$. This allows prolonged retention of demulcents on the damaged ocular surface. ${ }^{23,24}$ HPG-containing eyedrops also decrease inflammatory response and friction and promote corneal epithelial healing by preferentially binding to hydrophobic zones in the tear film, forming a protective layer that leads to tear-film stabilization. ${ }^{23-26}$

Use of a combination of different polymers, such as HPG and HA, in a single artificial-tear formulation can be useful, as it potentially synergizes the properties of the separate polymers and improves ocular bioavailability of the active ingredients, which may result in enhanced ocular surface benefits to dry-eye patients. ${ }^{22}$ Most artificial tears, particularly those that are saline-based, have short retention time and poor corneal wettability and thus require frequent dosing. As the treatment aim in DED is to improve patient quality of life, it is desirable to have tear substitutes that can provide prolonged hydration and lubrication of the ocular surface and thus reduce the frequency of instillations.
Dual-polymer HPG-HA artificial tears, built on the PEG/PG-HPG platform, combine the therapeutic properties of HPG and HA in a single formulation that may provide greater ocular surface hydration and lubrication and decrease friction compared to a single polymer-based artificial-tear formulation. ${ }^{27}$ The HPG-HA dual-polymer formulation demonstrated significantly greater hydration protection, lubrication, cell viability, and better cell-barrier function than the individual polymers in an in vitro study conducted in human corneal epithelium models. ${ }^{27}$ Compared with other HA-containing single- and dual-polymer-based artificial tear formulations, mouse corneas treated with the HPG-HA formulation demonstrated faster wound healing within 24 hours of injury, indicating the potential benefit of this formulation to promote corneal reepithelialization and restoration of ocular surface health in patients with DED. ${ }^{28}$

Although there was a reduction in ocular staining based on the sequential testing strategy, the study did not meet any of the secondary end points and HPG-HA did not show any improvement over SH in terms of IDEEL treatmentsatisfaction scores or TFBUT. The higher IDEEL treatment inconvenience noted with the HPG-HA formulation could have been related to the transient blur in vision that some patients experience immediately upon instillation of artificial tears with higher viscosity, in this case a dual polymer, compared with single-polymer lubricant drops. The blur may be due to the longer retention of HPG upon instillation of the formulation, which cross-links and improves the integrity of the tear film. ${ }^{7}$ Patients with dry eye often complain of visual discomfort, which is attributed to unstable tear-film and decreased blink rate. Artificial tear formulations with higher viscosity have an increased residence time on the ocular surface..$^{29,30}$ Cagini et al observed that the use of a higher-viscosity cross-linked HA tear substitute resulted in marked improvement in topographical indices in DED patients compared to those treated with HA alone 30 and 60 minutes after instillation. ${ }^{30} \mathrm{PEG} / \mathrm{PG}-\mathrm{HPG}$ artificial tears have also been shown to significantly prolong TFBUT at 45, 60, and 90 minutes after instillation, improve the ocular protection index up to 90 minutes after instillation, retard visual acuity decay between blinks at 90 minutes after instillation, and have a beneficial effect on functional vision in dry-eye patients. ${ }^{6,31,32}$ The IDEEL treatment-inconvenience questionnaire has only four items, and it is possible that the question related to blurriness after using eyedrops could have driven lower IDEEL scores in the HPG-HA group, but it does not capture information related to ocular comfort that patients may eventually experience in the longer run. 
Blurred vision was the more frequently reported ocular AE in HPG-HA group than in the SH group. Overall, the HPG-HA formulation was well tolerated, and no meaningful differences were observed in the rate of ocular treatmentemergent AEs between the two groups. No untoward safety signals or trends were observed with use of the dual-polymerbased HPG-HA formulation in patients with DED.

This study did not assess improvement in IDEEL symptom-bother score or symptoms in the two treatment groups. This would have been a useful parameter to distinguish the clinical benefit of using dual-polymer-based lubricant drops, since patients with DED primarily seek treatment because of ocular discomfort. Also, since DED is a complex multifactorial condition with signs and symptoms often uncorrelated, there are no standard criteria to determine the therapeutic efficacy of different treatments or to compare treatment outcomes. ${ }^{33}$

\section{Conclusion}

The dual-polymer-based HPG-HA artificial-tear formulation was noninferior to the single-polymer $\mathrm{SH}$ formulation in terms of improvement in ocular staining in patients with DED. No new safety findings were identified outside the known profile of other HPG-containing lubricant eyedrops.

\section{Data sharing statement}

The study results are available at https://ClinicalTrials. gov/ct2/show/results/NCT02470429?term=NCT02470429 \&rank $=1$. The data sets generated and/or analyzed during the current study are available from the study sponsor on reasonable request.

\section{Acknowledgments}

Manuscript-writing support was provided by Shivani Vadapalli (Novartis Healthcare Pvt. Ltd, Hyderabad, India). This study was sponsored by Alcon Research Ltd, Fort Worth, Texas, USA (now Novartis Pharmaceuticals Corporation).

\section{Disclosure}

ML is an occasional consultant and has received honoraria from Alcon, Allergan, Dompé, Horus Pharma, MSD, Novartis, Santen, Shire, and Théa and research support from Théa. CB is a consultant and has received honoraria from Allergan, Dompé, Horus Pharma, Novartis, Santen, and Théa and research support from Horus Pharma, Santen, and Théa. DG is a consultant and received honorarium from Novartis, Théa, and Shire. MG's institute received funding support from Alcon for the conduct of this study. AO is a former employee of Novartis Pharmaceuticals. SS and DB have no potential conflicts of interest.

\section{References}

1. The definition and classification of dry eye disease: report of the Definition and Classification Subcommittee of the International Dry Eye WorkShop (2007). Ocul Surf. 2007;5(2):75-92.

2. Management and therapy of dry eye disease: report of the Management and Therapy Subcommittee of the International Dry Eye WorkShop (2007). Ocul Surf. 2007;5(2):163-178.

3. Messmer EM. The pathophysiology, diagnosis, and treatment of dry eye disease. Dtsch Arztebl Int. 2015;112(5):71-82.

4. Lemp MA. Management of dry eye disease. Am J Manag Care. 2008; 14(3 Suppl):S88-S101.

5. Al-Saedi Z, Zimmerman A, Bachu RD, et al. Dry eye disease: present challenges in the management and future trends. Curr Pharm Des. 2016;22(28):4470-4490.

6. Torkildsen G. The effects of lubricant eye drops on visual function as measured by the inter-blink interval visual acuity decay test. Clin Ophthalmol. 2009;3:501-506.

7. Benelli U. Systane lubricant eye drops in the management of ocular dryness. Clin Ophthalmol. 2011;5:783-790.

8. Labetoulle M, Messmer EM, Pisella PJ, Ogundele A, Baudouin C. Safety and efficacy of a hydroxypropyl guar/polyethylene glycol/ propylene glycol-based lubricant eye-drop in patients with dry eye. Br J Ophthalmol. 2017;101(4):487-492.

9. Jacobi C, Kruse FE, Cursiefen C. Prospective, randomized, controlled comparison of SYSTANE UD eye drops versus VISINE INTENSIV $1 \%$ EDO eye drops for the treatment of moderate dry eye. $J$ Ocul Pharmacol Ther. 2012;28(6):598-603.

10. Baudouin C, Galarreta DJ, Mrukwa-Kominek E, et al. Clinical evaluation of an oil-based lubricant eyedrop in dry eye patients with lipid deficiency. Eur J Ophthalmol. 2017;27(2):122-128.

11. Christensen MT, Cohen S, Rinehart J, et al. Clinical evaluation of an HP-guar gellable lubricant eye drop for the relief of dryness of the eye. Curr Eye Res. 2004;28(1):55-62.

12. Hartstein I, Khwarg S, Przydryga J. An open-label evaluation of HPGuar gellable lubricant eye drops for the improvement of dry eye signs and symptoms in a moderate dry eye adult population. Curr Med Res Opin. 2005;21(2):255-260.

13. Nakamura M, Hikida M, Nakano T, Ito S, Hamano T, Kinoshita S. Characterization of water retentive properties of hyaluronan. Cornea. 1993; 12(5):433-436.

14. Gomes JA, Amankwah R, Powell-Richards A, Dua HS. Sodium hyaluronate (hyaluronic acid) promotes migration of human corneal epithelial cells in vitro. Br J Ophthalmol. 2004;88(6):821-825.

15. Mochizuki H, Yamada M, Hato S, Nishida T. Fluorophotometric measurement of the precorneal residence time of topically applied hyaluronic acid. Br J Ophthalmol. 2008;92(1):108-111.

16. Dogru M, Nakamura M, Shimazaki J, Tsubota K. Changing trends in the treatment of dry-eye disease. Expert Opin Investig Drugs. 2013; 22(12):1581-1601.

17. Schmidl D, Schmetterer L, Witkowska KJ, et al. Tear film thickness after treatment with artificial tears in patients with moderate dry eye disease. Cornea. 2015;34(4):421-426.

18. Brjesky VV, Maychuk YF, Petrayevsky AV, Nagorsky PG. Use of preservative-free hyaluronic acid (Hylabak) for a range of patients with dry eye syndrome: experience in Russia. Clin Ophthalmol. 2014;18:1169-1177.

19. Aragona P, Papa V, Micali A, Santocono M, Milazzo G. Long term treatment with sodium hyaluronate-containing artificial tears reduces ocular surface damage in patients with dry eye. Br J Ophthalmol. 2002; 86(2):181-184.

20. Prabhasawat P, Tesavibul N, Kasetsuwan N. Performance profile of sodium hyaluronate in patients with lipid tear deficiency: randomised, double-blind, controlled, exploratory study. Br J Ophthalmol. 2007; 91(1):47-50. 
21. Johnson ME, Murphy PJ, Boulton M. Effectiveness of sodium hyaluronate eyedrops in the treatment of dry eye. Graefes Arch Clin Exp Ophthalmol. 2006;244(1):109-112.

22. Zambito Y, Di Colo G. Polysaccharides as excipients for ocular topical formulations. Biomater Appl Nanomed. 2011:253-290.

23. Springs C. Novel ocular lubricant containing an intelligent delivery system: details of its mechanism of action. Dev Ophthalmol. 2010;45:139-147.

24. Ubels JL, Clousing DP, van Haitsma TA, et al. Pre-clinical investigation of the efficacy of an artificial tear solution containing hydroxypropylguar as a gelling agent. Curr Eye Res. 2004;28(6):437-444.

25. Meyer AE, Baier RE, Chen H, Chowhan M. Differential tissue-on-tissue lubrication by ophthalmic formulations. J Biomed Mater Res B Appl Biomater. 2007;82(1):74-88.

26. Epstein SP, Gadaria-Rathod N, Wei Y, Maguire MG, Asbell PA. HLA-DR expression as a biomarker of inflammation for multicenter clinical trials of ocular surface disease. Exp Eye Res. 2013;111:95-104.

27. Rangarajan R, Kraybill B, Ogundele A, Ketelson HA. Effects of a hyaluronic acid/hydroxypropyl guar artificial tear solution on protection, recovery, and lubricity in models of corneal epithelium. J Ocul Pharmacol Ther. 2015;31(8):491-497.

28. Ogundele A, WWY K, Carlson E. Evaluating the effect of hyaluronic acid-containing artificial tear products on corneal wound reepithelialization in an in vivo study. ARVO 2017 Annual Meeting Abstract. Available from: http://www.arvo.org/webs/am2017/sectionpdf/CO/ Session $\% 20107 \% 20$ Cornea $\% 20$ Wound $\% 20$ Healing $\% 20$ and $\% 20$ Repair.pdf
29. Paugh JR, Nguyen AL, Ketelson HA, Christensen MT, Meadows DL. Precorneal residence time of artificial tears measured in dry eye subjects. Optom Vis Sci. 2008;85(8):725-731.

30. Cagini C, Torroni G, Fiore T, et al. Tear film stability in Sjögren syndrome patients treated with hyaluronic acid versus crosslinked hyaluronic acid-based eye drops. J Ocul Pharmacol Ther. 2017;33(7): $539-542$.

31. Guillon M, Maissa CA, Wong S, Griffin JM, Christensen MT. Functional visual performance of systane ultra after 4 weeks of use. Invest Ophthalmol Vis Sci. 2011;52: ARVO Annual Meeting E-Abstract 3834. Available from: http://iovs.arvojournals.org/article. aspx?articleid=2355981

32. D'Arienzo P, Ousler GW, Schindelar MS. A comparison of two marketed artificial tears in improvement of tear film stability as measured by tear film break-up time (TFBUT) and ocular protection index (OPI). Poster presented at: Tear Film and Ocular Surface Society Meeting; 2007; Taormina, Sicily.

33. Sullivan BD, Crews LA, Messmer EM, et al. Correlations between commonly used objective signs and symptoms for the diagnosis of dry eye disease: clinical implications. Acta Ophthalmol. 2014;92(2): $161-166$. 


\section{Supplementary materials Ocular staining}

To determine total ocular surface staining, a single drop of preservative-free saline was placed on a sodium fluorescein strip and the saline allowed to saturate the tip of the strip fully. The patient's lower eyelid was retracted and the saline-moistened strip gently placed onto the lower tarsal conjunctiva. Instillation time was recorded. The patient was then asked to blink several times. Within 30 seconds of instillation of the stain, the right ocular, nasal, and temporal conjunctiva were examined using a slit lamp with a cobalt blue exciter and a Wratten yellow filter. The upper eyelid was then lifted slightly to allow examination of the entire corneal surface. The starting time of the examination was recorded. To the extent possible, TOSS was determined by the same investigator for the same patient using the same slit lamp with the same settings at each study visit.

\section{IDEEL treatment-satisfaction (effectiveness and inconvenience) questionnaires}

The impact of dry eye on everyday life (IDEEL) treatmentsatisfaction questionnaire was completed on days 0,15 , and 42 and consisted of ten items that assessed each patient's general satisfaction with treatment use over the 2 weeks before completing the questionnaire. Question 1 asked patients to report how frequently they used dry-eye treatment, with responses ranging from "none of the time" (equating to a score of 0 ) to "all of the time" (equating to a score of 4). Questions 2-6 asked patients, using a 0-4 scale, to rate how often certain statements were true (eg, "I was happy with how quickly my treatments worked"). Question 7 asked (yes/no) "Do you ever use eyedrops to treat your dry eye?" Patients who answered yes to this question were then asked to rate ( $0-4$ scale) how often they experienced each of three situations (eg, "I was bothered by blurriness shortly after using my eyedrops"). For all items, patients were instructed to select a single response. The first IDEEL score was calculated as the

Clinical Ophthalmology

\section{Publish your work in this journal}

Clinical Ophthalmology is an international, peer-reviewed journal covering all subspecialties within ophthalmology. Key topics include: Optometry; Visual science; Pharmacology and drug therapy in eye diseases; Basic Sciences; Primary and Secondary eye care; Patient Safety and Quality of Care Improvements. This journal is indexed on

Submit your manuscript here: http://www.dovepress.com/clinical-ophthalmology-journal mean response to questions $2-5$, with the result multiplied by 25 . The score was calculated as long as at least two of the four questions were answered. The second IDEEL score was then calculated as the mean response to questions 6,8 , 9 , and 10 , with the result multiplied by 25 . Again, the score was calculated as long as at least two of the four questions were answered. Both first and second IDEEL scores were recorded. Responses to questions 1 and 7 were not included in the score computations. The possible range of each IDEEL score was 0 (complete disability) to 100 (no disability).

\section{Tear-film breakup time}

Tear-film breakup time was assessed using a sodium fluorescein strip. The tip of the strip was allowed gently to touch the inferior bulbar conjunctival surface to cover the inferior bulbar conjunctiva generously with fluorescein stain. The patient was instructed to blink three times and then stare without blinking. The investigator monitored the integrity of the tear film by using a slit lamp, and measured the time from the last blink using a stopwatch until one or more dry spots had appeared in precorneal tear film.

\section{Tear osmolarity}

Tear-osmolarity measurements were obtained using TearLab osmolarity readers, pens, and test cards. Measurement was conducted by obtaining one reading from each eye, starting with the right eye. Without pulling down the lower lid, the tip of the pen was lowered onto the tear lake at the lower margin. Care was taken not to touch the lashes in an effort to avoid stimulation of reflex tearing. If reflex tearing did occur, the investigator was to remove the pen from the eye, wait 15 minutes, and then attempt a repeat sample by using a new test card. A maximum of three attempts were allowed.

\section{Tear-film kinetic measures and objective bulbar conjunctival staining}

These were conducted in accordance with the instruction manual, only at selected study centers.

\section{Dovepress}

PubMed Central and CAS, and is the official journal of The Society of Clinical Ophthalmology (SCO). The manuscript management system is completely online and includes a very quick and fair peer-review system, which is all easy to use. Visit http://www.dovepress.com/ testimonials.php to read real quotes from published authors. 